\title{
Implementation of the reaching every district (RED) approach: experience from North Sudan
}

\author{
T.K. Ryman, ${ }^{7}$ E.A. Elsayed, ${ }^{2}$ A.A.-M. Mustafa, ${ }^{2}$ N.M. Widaa, ${ }^{2}$ A. Omer $^{2}$ and R. Kamadjeu ${ }^{7}$
}

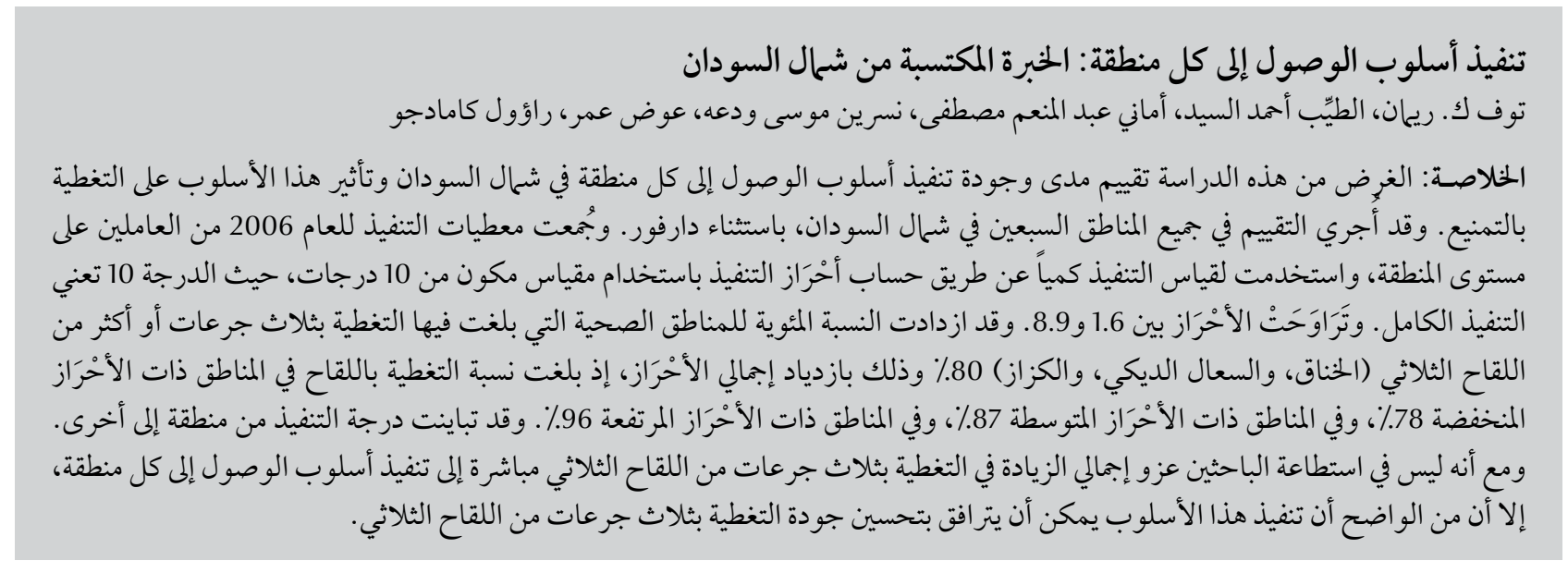

ABSTRACT The purpose of this evaluation was to assess the extent and quality of implementing the Reaching Every District (RED) approach in North Sudan and its impact on immunization coverage. The evaluation was conducted in all 70 districts of North Sudan, excluding Darfur. District RED implementation data for 2006 were collected from district staff and used to quantify implementation by calculating Implementation Scores (IS) using a 10-point scale, with 10 being fully implemented. Overall RED IS ranged from 1.6 to 8.9. The percentage of districts with diphtheria-pertussis-tetanus (DPT) 3 coverage $\geq 80 \%$ increased as the overall RED IS increased, $78 \%, 87 \%$, and $96 \%$ in low-, medium-, and high-scoring groups respectively. The degree of RED implementation varied across districts. Although it is not possible to directly attribute the overall increase in DPT3 coverage to RED implementation, RED implementation quality might be associated with improved DPT3 coverage.

\section{Mise en œuvre de l'approche «Atteindre chaque district » : expérience au nord du Soudan}

RÉSUMÉ L'objectif de la présente évaluation était de mesurer l'étendue et la qualité de la mise en œuvre de l'approche «Atteindre chaque district » dans le nord du Soudan et ses effets sur la couverture vaccinale. L'évaluation a été conduite dans l'ensemble des 70 districts du nord du Soudan, à l'exception du Darfour. Les données relatives à la mise en œuvre de l'approche « Atteindre chaque district » pour 2006 ont été recueillies par l'équipe de district et exploitées pour quantifier la mise en œuvre en calculant les scores correspondants à l'aide d'une échelle en dix points. Le score de dix correspondait à une mise en œuvre complète. Les scores globaux concernant la mise en œuvre de l'approche «Atteindre chaque district» allaient de 1,6 à 8,9. Le pourcentage de districts couverts à $80 \%$ ou plus par la troisième dose du vaccin antidiphtérique-antitétanique-anticoquelucheux (DTC-3) avait augmenté, comme les scores de mise en œuvre globaux qui atteignaient $78 \%$, $87 \%$, et $96 \%$ dans les groupes aux scores faible, moyen et élevé, respectivement. La progression de la mise en œuvre de l'approche était variable en fonction des districts. Il n'est pas possible de créditer directement l'augmentation globale de la couverture par le vaccin DTC-3 à la mise en œuvre de l'approche «Atteindre chaque district», mais la qualité de la mise en oeuvre a pu contribuer à l'amélioration de la couverture vaccinale par ce vaccin. 


\section{Introduction}

The Reaching Every District (RED) routine immunization approach aims to improve immunization services in order to guarantee sustainable and equitable immunization for every child. RED is a key strategy for the World Health Organization (WHO) and the United Nations Children's Fund (UNICEF) as well as a method for achieving the Global Immunization Vision and Strategy (GIVS) goals for increasing and maintaining coverage of at least $90 \%$ nationally and at least $80 \%$ in every district by 2010 [1].

RED was developed in 2002 in the African Region and consists of 5 strategies: 1) re-establishing outreach; 2) supportive supervision; 3 ) monitoring and use of data for action; 4) strengthening planning and management of resources through micro-plans; and 5) increasing community links [2]. RED was designed to empower district level health authorities and immunization staff to plan, implement, and monitor interventions and activities to improve immunization services.

As of 2006, 53 countries had started implementing some components of RED [3], yet systematic evaluations of RED implementation at national and district levels were limited. Furthermore, there were no comparisons of the quality of RED implementation with vaccination coverage. In 2005, a 5-country evaluation of RED implementation was conducted in Africa; however, the convenience sample of districts visited was small and the evaluation did not compare the extent of implementation between districts [4].

Interventions to improve immunization coverage in North Sudan were implemented with success between 1994 and 1996 [5]. However, in 2002, North Sudan still had more than 50\% of districts with third dose diphtheriapertussis-tetanus vaccine (DPT3) coverage below the Global Immunization
Vision and Strategy target of $80 \%$, making it desirable to implement RED. North Sudan was selected for evaluation because it had been implementing RED for more than 4 years. In 2003, North Sudan began phasing in RED implementation in medium-performing districts based on DPT3 coverage, followed by high- and low-performing districts. By the end of 2003, all districts were implementing RED.

In North Sudan, RED implementation began with the creation of federal level guidelines for routine immunization planning and supervision. RED trainings were conducted with the support of WHO and GAVI Alliance Immunization Services Support (ISS) funding. States and districts provided necessary resources for all routine immunization activities. However, based on the micro-plans, districts could receive additional Global Immunization Vision and Strategy Alliance funds from the federal Expanded Programme on Immunization (EPI) to support supervisory visits (e.g. transportation, per diems) and expenses for outreach and mobile sessions. When necessary, additional resources, such as vehicles and cold chain equipment, were also provided by the federal EPI.

This evaluation was conducted to document North Sudan's experience in implementing RED including the extent and quality of RED implementation, lessons learned, and potential impact on immunization coverage. Findings of the evaluation will help strengthen RED within North Sudan as well as provide lessons for other countries.

\section{Methods}

An evaluation of RED was conducted from February to July 2007, in 70 of the 93 districts of North Sudan; the 23 districts of Darfur were excluded due to security issues. Two primary data collection methods were used: 1 ) worksheets for district-level staff in all study districts $(n=70)$ and 2) semistructured interview questionnaires for in-depth case studies in 4 selected districts. Immunization coverage and drop-out data from 2006 were obtained from administrative sources. Districts were categorized as achieving $80 \%$ DPT3 coverage or not for purposes of categorical data analysis.

\section{Creating RED implementation scores}

Worksheets completed byeach district's routine immunization manager, known in North Sudan as "Locality Officers" (LOs) were used to measure both the extent and quality of RED implementation during 2006, which was the most recent year with complete data available. Two types of data collection worksheets were developed; 1) a general assessment worksheet self completed by district-level staff and 2) a worksheet to guide a peer in reviewing district level immunization documents.

The self-completed general assessment worksheets included objective questions on each of the 5 RED components and contextual factors that could have significantly impacted RED implementation or immunization outcomes (e.g. staff turnover, transportation, geography, on-going conflict, funding). In February 2007, LOs from the 70 districts were given the general assessment worksheet and requested to bring the completed worksheet to the next review meeting in March 2007. The LOs were also instructed to bring to the March meeting district-level documents and data (e.g. supervision records, wall monitoring charts, immunization micro-plans, refrigerator temperature monitoring charts) for peer-review, in order to minimize self-reporting bias. Each LO shared their supplemental materials with a peer LO sitting adjacent and a facilitator led a review and systematic rating of the supplemental documents; findings were recorded on the peerreview worksheet. 
A rating system, standard across all districts, was used to score each RED component by summing the indicators from the general assessment and peerreview worksheets. Indicators were assigned a weight by an expert opinion team prior to RED component score calculation. The implementation quality indicators collected from the selfreported and peer-review worksheets and their respective weights are shown in Table 1.

An overall RED Implementation Score (IS) was calculated for each district by summing the 5 RED component scores, with each RED component equally weighted. The overall RED IS, is on a 10-point scale, with 10 being fully implemented. Three "RED implementation" groups were formed by splitting the districts into thirds by RED IS: low $(\leq 5.28)$, medium (5.28-6.31) and high $(\geq 6.31)$.

\section{In-depth case studies}

The evaluation team selected 4 districts for in-depth case studies of RED implementation based on use of acceleration activities and RED IS coverage. To gain perspective from a range of districts, 2 of the 4 selected districts had acceleration activities to increase lagging mid-year coverage (1 with a high and one with low RED IS), and 2 districts did not have acceleration activities to increase coverage (1 with a high RED IS and 1 with a low RED IS). Districts were excluded if they could not be visited because of conflict or if they had implemented RED for less than 1 year (i.e. recently subdivided districts).

In-depth case study data were collected at the district EPI office and at the 2 health centres within the district with the highest and the lowest performance, based on 2006 administrative DPT3 immunization coverage. Data were collected using a semi-structured interview questionnaire, which also included the data which had been collected during the general assessment in order to assess data validity. Results were compared between districts and reported as anecdotal evidence. To avoid reporting bias, visits were conducted by Arabic-speaking short-term consultants from the WHO who did not work in the district and the US Centers for Disease Control and Prevention (CDC) staff accompanied by a Sudanese counterpart familiar, but not affiliated, with the EPI programme. All data collectors were familiar with the North Sudan EPI programme.

\section{Data analysis}

The main outcome measure for this evaluation was immunization coverage [i.e. DPT first dose (DPT1), DPT3, and measles vaccine]. District and national immunization coverage was calculated by dividing the district and national cumulative doses administered by district and national denominators (projected census data). Changes in immunization coverage between 2002 and 2006 were analysed using $t$-tests with the null hypothesis being no change in coverage for this period. Some analyses were stratified based on whetherin 2006 districts used accelerated immunization activities; intensive efforts, funded by the federal EPI programme to raise immunization coverage in under-performing districts after the mid-year review, since this may influence immunization coverage, our evaluation outcome. In 2006, based on federal records, half of all districts used accelerated immunization activities.

An additional outcome measure used in this evaluation was drop-out, the percent of children starting but not completing the immunization series. Drop-out was calculated both from DPT 1 to DPT3 and from DPT 1 to measles vaccine; acceptable drop-out is between $0 \%$ and $10 \%$. Both calculations were analysed to measure the proportion of children that drop out at different ages (DPT3 is given at 14 weeks and measles vaccine is given at 9 months). Drop-outs are traced through defaulter tracing systems, and children subsequently vaccinated are considered to have been recouped.

Data cleaning and analysis were conducted by CDC. Quantitative data analysis was performed using SAS (Statistical Analysis Software), version 9.1. Pearson correlation coefficients were calculated between RED IS (i.e. overall, component, individual RED continuous indicators), contextual factors (e.g. political commitment, management, acceleration activities) and immunization coverage (i.e. DPT1, DPT3, measles vaccine). A paired $t$-test was used to test for a significant increase in performance indicators from 2002 to 2006. Exact chi-squared test and exact chi-squared test for trend were used to analyse categorical variables. Statistical significance was defined as a $P$-value $<0.05$. Qualitative data collected during the in-depth case study interviews were analysed for common themes.

\section{Results}

\section{Immunization coverage 2002-06}

National DPT1, DPT3 and measles coverage in North Sudan increased $5 \%, 13 \%$ and $7 \%$ respectively between 2002 and 2006 (Table 2). In the 52 districts with the same boundaries for both years, the increase in coverage was statistically significant for all 3 vaccine coverage measures (paired $t$-test, $P<$ 0.05) (Table 2). In 2002, 27 (43\%) of 62 districts in North Sudan (excluding Darfur) had achieved DPT3 coverage levels $\geq 80 \%$ compared to $61(81 \%)$ of 70 accessible districts in $2006\left(\chi^{2}=28.1\right.$, $P<0.001)$. There were 8 additional districts in 2006 because of district subdividing. National measles vaccine coverage (78\%) was lower than DPT3 coverage (90\%) in 2006. Although coverage between 2002 and 2006 increased for DPT1, DPT3 and measles, the increase was not constant each year 


\begin{tabular}{|c|c|c|c|}
\hline Component indicator & Individual indicator & $\begin{array}{l}\text { Individual } \\
\text { indicator weight }^{\mathrm{a}}\end{array}$ & $\begin{array}{l}\text { Level for achieving } \\
\text { high RED IS }\end{array}$ \\
\hline \multirow[t]{3}{*}{ Outreach $(n=3)$} & $\%$ of planned outreach sessions held & 0.33 & $\geq 90 \%$ \\
\hline & $\%$ of planned mobile sessions held & 0.33 & $\geq 90 \%$ \\
\hline & $\%$ of planned fixed sessions held & 0.33 & $\geq 95 \%$ \\
\hline \multirow[t]{10}{*}{ Supervision $(n=10)$} & $\%$ of planned supervision to fixed sites by LO & 0.10 & $\geq 80 \%$ \\
\hline & $\begin{array}{l}\% \text { of fixed sites receiving } \geq 1 \text { supervisory visit during the } \\
\text { year }\end{array}$ & 0.10 & $\geq 90 \%$ \\
\hline & $\begin{array}{l}\text { No. of monthly locality supervision reports submitted } \\
\text { to state }\end{array}$ & 0.10 & 12 \\
\hline & $\begin{array}{l}\text { No. of supervision visits the locality received from the } \\
\text { state }\end{array}$ & 0.10 & $\geq 5$ \\
\hline & $\begin{array}{l}\text { No. of supervision visits the locality received from the } \\
\text { federal }\end{array}$ & 0.10 & $\geq 3$ \\
\hline & $\%$ of outreach teams visited by $L O$ & 0.10 & $\geq 10 \%$ \\
\hline & $\%$ of mobile teams visited by $\mathrm{LO}$ & 0.10 & $\geq 10 \%$ \\
\hline & Records available for all supervisory visits & 0.10 & Yes \\
\hline & $\begin{array}{l}\text { Quality of supervision records (as per peer-review } \\
\text { guidelines) }\end{array}$ & 0.10 & $100 \%$ \\
\hline & $\begin{array}{l}\text { Quality supervision records (as per peer-review } \\
\text { guidelines) }\end{array}$ & 0.10 & $100 \%$ \\
\hline \multirow[t]{11}{*}{$\begin{array}{l}\text { Monitoring for action } \\
(n=11)\end{array}$} & $\begin{array}{l}\text { Monitoring charts available for all locality \& all fixed } \\
\text { sites }\end{array}$ & 0.09 & Yes \\
\hline & Monitoring chart with no graphing errors & 0.09 & Yes \\
\hline & Monitoring chart with no calculation errors & 0.09 & Yes \\
\hline & $\%$ of fixed sites with defaulter tracking for the entire year & 0.09 & $\geq 95 \%$ \\
\hline & Defaulter tracking success rate (recoup) & 0.09 & $>80 \%$ \\
\hline & $\%$ of fixed sites sending complete monthly reports & 0.09 & $100 \%$ \\
\hline & $\%$ of fixed sites sending timely monthly reports & 0.09 & $100 \%$ \\
\hline & No. of review meeting with fixed sites planned & 0.09 & 12 \\
\hline & $\%$ of review meetings with fixed sites held & 0.09 & $\geq 75 \%$ \\
\hline & No. of monthly state level review meeting LO attended ${ }^{c}$ & 0.09 & 12 \\
\hline & $\begin{array}{l}\text { All cold chain equipment monitored twice daily (except } \\
\text { Friday) }\end{array}$ & 0.09 & Yes \\
\hline \multirow[t]{13}{*}{$\begin{array}{l}\text { Planning \& management } \\
\text { of resources }(n=10)\end{array}$} & $\begin{array}{l}\text { Completeness of micro-plan (as per peer-review } \\
\text { guidelines) }\end{array}$ & 0.20 & $100 \%$ \\
\hline & $\%$ of locality refrigerators functioning through the year & 0.10 & $\geq 90 \%$ \\
\hline & Two trainings completed by LO & 0.10 & Yes \\
\hline & $\%$ of vaccinators fully trained & 0.10 & $100 \%$ \\
\hline & $\begin{array}{l}\text { Generator in locality office functional throughout the } \\
\text { year }\end{array}$ & 0.10 & Yes \\
\hline & Vaccine stock-out & 0.05 & 0 \\
\hline & Vaccine stock-out duration & 0.05 & 0 \\
\hline & Syringe stock-out & 0.05 & 0 \\
\hline & Syringe stock-out duration & 0.05 & 0 \\
\hline & Tally sheet stock-out & 0.05 & 0 \\
\hline & Tally sheet stock-out duration & 0.05 & 0 \\
\hline & Vaccine card stock-out & 0.05 & 0 \\
\hline & Vaccine card stock-out duration & 0.05 & 0 \\
\hline
\end{tabular}




\begin{tabular}{|c|c|c|c|}
\hline Component indicator & Individual indicator & $\begin{array}{l}\text { Individual } \\
\text { indicator weight }^{2}\end{array}$ & $\begin{array}{l}\text { Level for achieving } \\
\text { high RED IS }\end{array}$ \\
\hline \multirow[t]{5}{*}{ Community links $(n=5)$} & $\begin{array}{l}\text { No. of different types of social mobilization activities } \\
\text { planned }\end{array}$ & 0.20 & $\geq 6$ \\
\hline & $\%$ of planned social mobilization activities implemented & 0.20 & $\geq 100 \%$ \\
\hline & EPI Friends Society member involvement & 0.20 & Yes \\
\hline & Women's Union member involvement & 0.20 & Yes \\
\hline & No. of other community groups involved in EPI & 0.20 & $\geq 2$ \\
\hline
\end{tabular}

aIndicators were assigned a weight by an expert opinion team, each component has a weight of 0.20 for the overall RED IS score.

${ }^{b}$ High level of implementation equalled approximately the top $3 \mathrm{rd}$ of districts, using natural cut-off points.

In 7 state only 6 meetings were held per year because of distances.

$R E D=$ reaching every district; $I S=$ implementation score; $E P I=$ Expanded Programme on Immunization; $L O=$ locality officer.

(Figure 1). National DPT1 to DPT3 drop-out decreased from $15 \%$ in 2002 to $6 \%$ in 2006 and has remained below the advised $10 \%$ level since 2003 (Table 2 ). The percentage of districts with a DPT1 to DPT3 drop-out of $0 \%-10 \%$ increased from $29 \%$ in 2002 to $59 \%$ in $2006\left(\chi^{2}=11.6, P<0.001\right)$. Nationally, DPT 1 to measles vaccine drop-out decreased from $22 \%$ in 2002 to $19 \%$ in 2006 (Table 2).

\section{RED IS and association with immunization coverage}

Overall IS were almost normally distributed with a mean of 5.7, a median of 5.7; district IS ranged from 1.6 to 8.9. Of the 5 RED components, the highest IS was for Monitoring for Action (6.9) and the lowest for Community Links (4.4) (Table 3).

The overall, component, or individual RED implementation indicators were not significantly correlated with DPT3 coverage based on calculating correlation coefficients [overall IS and DPT3 $(r=0.09, P=0.42)]$. The percentage of districts with DPT3 coverage $\geq$ $80 \%$ increased as the overall RED IS score increased, $78 \%, 87 \%$, and $96 \%$ in low-, medium- and high-scoring groups respectively (exact $\chi^{2}$-test for trend, $P$ $=0.058)$ (Table 3$)$. DPT3 coverage $\geq$ $80 \%$ was achieved by $77 \%$ of districts with low Community Links IS, 86\%

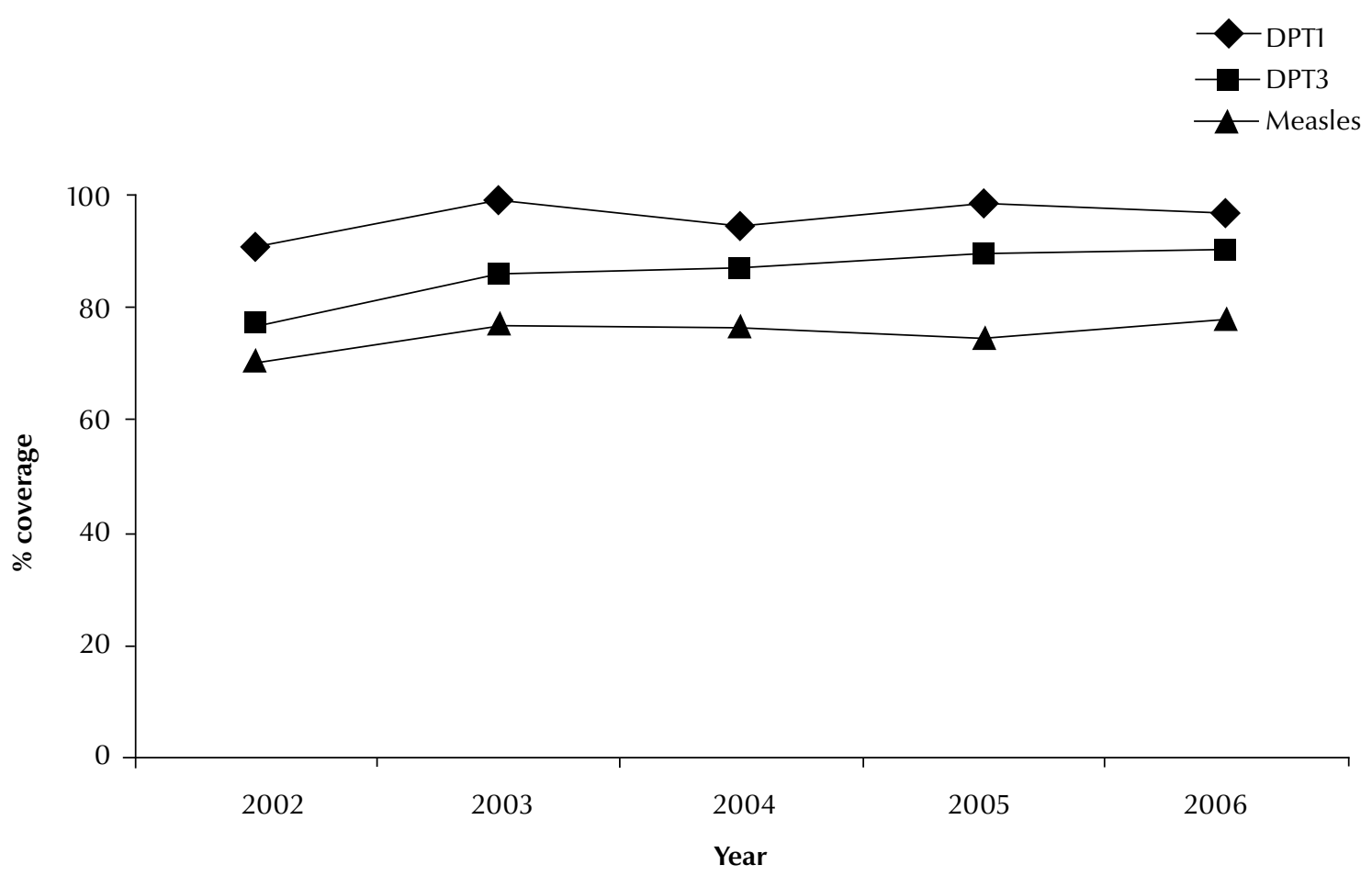




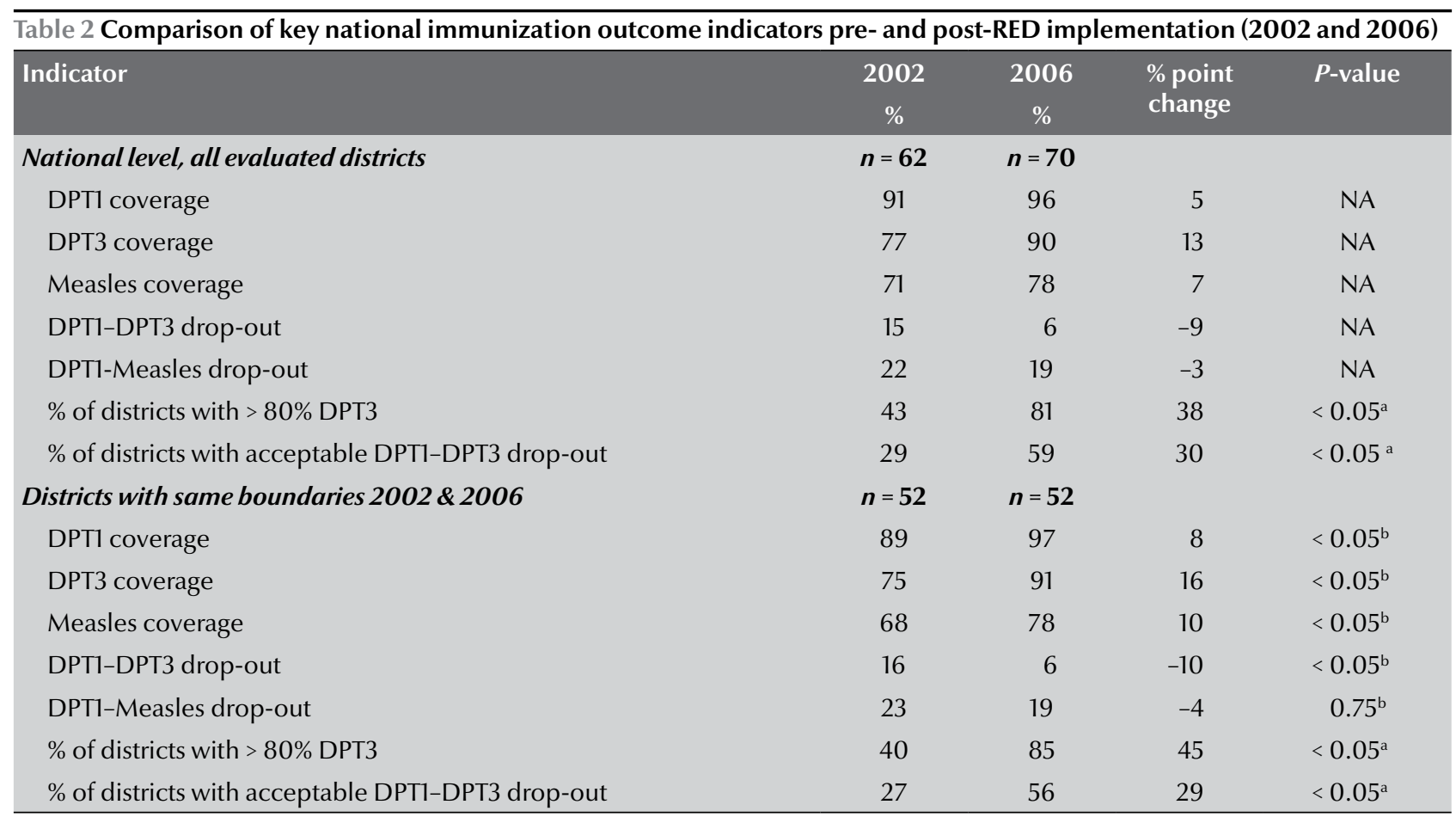

${ }^{a}$ Chi-squared test assessing the change in proportion of districts.

${ }^{b}$ Paired t-test assessing the change in coverage per districts 2002 and 2006.

RED = reaching every district; NA = not applicable because not all districts had the same boundaries in 2002 and 2006; DPT=diphtheria-pertussis-tetanus.

of districts with medium scores, and $100 \%$ of districts with high scores $(P$ $=0.01)($ Table 3$)$. None of the other RED components were significantly associated with DPT3 coverage.

\section{Findings by RED component \\ Outreach}

In $2006,35 \%$ of districts conducted $\geq$ $90 \%$ of planned mobile sessions, $45 \%$ of districts conducted $\geq 90 \%$ of planned outreach sessions, and $64 \%$ of districts conducted $\geq 90 \%$ of planned fixed immunization sessions. In case study sites, session plans were reported to be based on perceived need, not on manpower resources. In all case study districts, annual adjustments were being made to the distribution of fixed, outreach and mobile sites. There was an overall expressed interest in creating additional fixed immunization sites as these were perceived to be more sustainable.

\section{Supervision}

In $2006,31 \%$ of districts conducted $\geq$ $80 \%$ of planned immunization supervisory visits. All districts used the WHO
Data Quality Self-assessment (DQS) supervisory tool to evaluate the immunization monitoring system [6]. However, the methods used for providing feedback and recommendations varied. The peer-review of supervisory records indicated that $53 \%$ of districts had written records for all supervisory visits. All case study districts, except the urban district, maintained visitor notebooks in which supervisory visits, feedback and follow-up on recommendations were documented. In case study districts, documented supervisory visits to fixed immunization sites occurred as frequently as twice monthly.

During the case study visits, health facility staff reported an increase in supervisory visits over time and increased respect for their supervisors. One LO commented that, "in the past, supervisors told you all the things you were doing wrong, now they work with you to solve problems". All districts, excluding one, received at least 1 supervisory visit from the state in 2006 and $82 \%$ of districts received at least 1 visit from federal EPI staff.
Eighty-three per cent of LOs attended all planned state review meetings in 2006 (meetings occur monthly in most states). The exchange of experiences and problem-solving approaches discussed were described as the most useful aspects of the meetings.

\section{Monitoring for action}

Staff from all districts, except 1, brought 2006 wall monitoring charts to the March 2007 review meetings for peerreview. The charts were of high quality (i.e. calculations correctly completed, graphed and up-to-date). In $34 \%$ of districts, separate wall monitoring charts were available for the district and each fixed site.

In half of the 70 districts, $\geq 95 \%$ of fixed immunization sites had conducted defaulter tracing for the previous 12 months. Most health centres visited during case study visits cross-checked defaulter lists with neighbouring health centres to determine if children were vaccinated elsewhere. District defaulter recoup rates varied from $0 \%$ to $100 \%$, with half of all districts reporting $<60 \%$ 


\begin{tabular}{|c|c|c|c|c|c|}
\hline RED component & Mean RED IS & RED IS category & $\begin{array}{l}\text { No. of } \\
\text { districts }^{\mathrm{a}}\end{array}$ & $\begin{array}{c}\% \text { of districts with } \geq 80 \% \\
\text { DPT3 }(n=70)\end{array}$ & $P$-value ${ }^{b}$ \\
\hline \multirow[t]{3}{*}{ Overall } & \multirow[t]{3}{*}{5.7} & $\operatorname{Low}(\leq 5.28)$ & 23 & 78 & \multirow{3}{*}{0.058} \\
\hline & & Medium (5.28-6.31) & 23 & 87 & \\
\hline & & High $(>6.31)$ & 24 & 96 & \\
\hline \multirow[t]{3}{*}{ Outreach } & \multirow[t]{3}{*}{5.4} & $\operatorname{Low}(\leq 3.5)$ & 27 & 81 & \multirow{3}{*}{0.120} \\
\hline & & Medium (3.5-7.0) & 22 & 86 & \\
\hline & & High (> 7.0) & 21 & 95 & \\
\hline \multirow[t]{3}{*}{ Supervision } & \multirow[t]{3}{*}{5.7} & Low $(\leq 4.5)$ & 24 & 88 & \multirow{3}{*}{0.348} \\
\hline & & Medium (4.5-6.5) & 25 & 92 & \\
\hline & & High $(>6.5)$ & 21 & 81 & \\
\hline \multirow[t]{3}{*}{ Monitoring for action } & \multirow[t]{3}{*}{6.9} & Low $(\leq 5.5)$ & 20 & 85 & \multirow{3}{*}{0.271} \\
\hline & & Medium (5.5-7.5) & 23 & 83 & \\
\hline & & High $(>7.5)$ & 27 & 93 & \\
\hline \multirow{3}{*}{$\begin{array}{l}\text { Planning \& management of } \\
\text { resources }\end{array}$} & \multirow[t]{3}{*}{6.1} & Low $(\leq 5.5)$ & 17 & 82 & \multirow{3}{*}{0.338} \\
\hline & & Medium (5.5-6.5) & 25 & 88 & \\
\hline & & High $(>6.5)$ & 28 & 89 & \\
\hline \multirow[t]{3}{*}{ Linking with communities } & \multirow[t]{3}{*}{4.4} & Low $(\leq 3.5)$ & 26 & 77 & \multirow{3}{*}{0.014} \\
\hline & & Medium (3.5-5.5) & 22 & 86 & \\
\hline & & High (> 5.5) & 22 & 100 & \\
\hline
\end{tabular}

${ }^{a}$ The number of districts varies as the districts that met the $33 \%$ and $66 \%$ cut-off points differed by component. ${ }^{b} P$-values are based on exact $\chi^{2}$ test for trend.

$R E D=$ reaching every district; $I S$ = implementation score; $D P T=$ diphtheria-pertussis-tetanus .

of defaulters recouped and $25 \%$ of districts recouping $\geq 80 \%$ of defaulters. The percentage of fixed sites in the district with defaulter tracing for at least 12 months was not associated with recoup rates $(r=0.22, P=0.08)$, although anecdotal observations in the 4 case-study districts visited suggested there may be a relation.

Fifty-seven per cent of districts reported 100\% completeness of monthly reporting of doses administered in 2006; $35 \%$ reported on time every month. Staff reported that review meetings were used to collect reports, which according to immunization staff reduced the likelihood of delayed reporting.

\section{Planning and management of re- sources}

All districts had micro-plans that were reviewed during the peer-review process. Micro-plans meeting all federal requirements were found in $78 \%$ of districts. The micro-planning process reportedly changed over time to include greater involvement of vaccinators. One LO indicated that planning prior to the additional distribution of GAVI Alliance funds by the federal EPI staff was "resource-based" whereas now it is "results-based".

In $28 \%$ of districts, all vaccinators including non-permanent vaccinators were reported to be fully trained. Fortysix per cent of districts reported a stockout at either the district or health facility level of any antigen during 2006. Vaccine stock-outs lasted up to 151 days (mean $=21$ days at the district level)

\section{Linking with communities}

In $83 \%$ of districts, all of the planned community activities were conducted. However only 12 (17\%) of 70 districts planned $\geq 5$ different types of community activities to support the immunization programme; activities included radio advertisements and community meetings. Two groups are known to support EPI activities in North Sudan, the EPI Friend's Society and the
Women's Union. These groups were reported to be actively supporting (as defined by the LO) EPI activities in 33 and 30 districts respectively.

\section{Contextual factors}

Qualitative analysis of case study data from districts was considered to assess contextual factors including transportation, funding, management, accelerated immunization activities, supplemental immunization activities for poliomyelitis and measles, conflict, geography and staffing. Most data on contextual factors were missing from the general assessment worksheets; as such qualitative analysis of case study data was conducted. The most common challenge reported by districts was lack of transportation. During the in-depth case studies, funding for immunization services was not reported to be a limiting factor. Our data did not demonstrate a relationship between federal funding received by the district or the cost per child immunized and 
outcomes (i.e. immunization coverage, drop-out, RED IS).

In 2 case study districts which used accelerated immunization activities, the activities were initiated after the midyear review meeting, where districts not on track to meet the annual vaccination target were identified. Both districts agreed that acceleration immunization activities should not be needed and were working to increase the number of fixed immunization sites because they viewed them as more sustainable (e.g. staffed by permanent vaccinators and cost-effective) than acceleration activities.

\section{Discussion}

This evaluation compared the quality and extent of RED implementation among the 70 districts of North Sudan. Although it is not possible to attribute increases in vaccine coverage to RED implementation, significant increases in DPT 1, DPT3, and measles vaccine coverage were reported between 2002 and 2006, which coincided with RED implementation. We also found a weak association between the overall quality of RED implementation and achieving at least $80 \%$ DPT3 coverage. This association was not consistent across all RED strategies; linking with communities was the only strategy that was significantly associated with achieving 80\% DTP3 coverage.

The national DPT1 to DPT3 and DPT 1 to measles drop-out rates were not found to be associated with RED implementation. Yet the percentage of districts achieving acceptable DPT 1 toDPT3 drop-out (0\%-10\%) increased $30 \%$ after RED implementation. In addition, national DPT1 to DPT3 drop-out reached an acceptable level (0\%-10\%) by 2006. However, the DPT1 to measles drop-out rate did not improve at the district-level and had not achieved the acceptable drop-out rate of $0 \%-10 \%$ as of 2006 (19\%).
Collection of contextual factors and use of in-depth case studies helped to understand the complexities of linking an intervention with immunization coverage, an outcome influenced by a multitude of other factors. A contextual factor possibly obscuring an association of RED implementation and increased coverage was the introduction of acceleration activities. The acceleration activities were initiated after the mid-year review meetings, yet RED implementation was not significantly associated with mid-year 2006 coverage (pre-acceleration activities) either. Another contextual factor identified was the importance of political commitment and support for immunizations. In districts with strong political commitment, immunization activities were implemented despite problems, such as funding delays. Furthermore, additional funds were available through the GAVI Alliance during the same period as RED implementation.

Possible reasons for not finding a significant association between RED implementation and increased DPT3 coverage, if one existed, include insufficient time for the intervention to have had an impact, inaccurate vaccine coverage data (administrative data), or indicators of immunization programme performance that were not appropriate or adequately robust. Validation of data collected from district staff through worksheets during the 4 case study visits found a high level of agreement. As this is the first time this type of methodology to evaluate RED implementation has been developed, no standard indicators of immunization programme performance were available, and a comprehensive approach was used to identify these indicators. Additional work is needed to identify the most appropriate indictors for each RED component. We also attempted to collect the same data from both 2006 and 2002, before RED implementation, to assess baseline immunization programme performance indicators. However, these baseline data were not available. It is unclear if maintenance of records improved after RED implementation or if data were lost over time. While there are limitations to using administrative data for analysis, such data are a recognized method of estimating vaccination coverage in the absence of populationbased probability sampling coverage estimates [7]. Furthermore, we have no reason to believe that there would be differential bias across districts. The use of the $t$-test to assess coverage over time assumes that there were no changes in the methods or accuracy of administrative immunization data collected between 2002 and 2006.

This evaluation methodology yielded many lessons for improving future evaluations. The post-hoc method for cut-off point allocation of implementation scores was appropriate in this situation because no standard existed. Now that a baseline has been established in North Sudan, cut-off points can be set a priori to measure changes over time. The peer-review process proved a useful way for district immunization programme managers to review and compare their data with other districts. This process can also be used to help identify problems early and solve them more quickly. We suggest using a similar but less labour-intensive peerreview method for ongoing monitoring. For this evaluation $>50$ indicators were collected. From these data, we developed a list of 10 indicators that resulted in a similar correlation with coverage to those obtained using the comprehensive list of indicators. We think this simplified list would be more appropriate for ongoing monitoring. Another advantage of the peer-review process is that it is a simple way of allowing managers to monitor numerous districts simultaneously.

Although federal level EPI activities were not explicitly evaluated as a part of this evaluation, several observations of the federal level EPI involvement in 
North Sudan were observed, which might be useful to other country programmes. High levels of federal oversight and involvement were present from the start; a phased implementation was initially used for the RED approach, with medium-performing districts targeted first. However, this approach was quickly abandoned as federal staffmonitoring their data noted that high-performing districts were not maintaining their high standards, as the medium-performing districts were receiving additional funds. Subsequently the RED approach was quickly scaled up and implemented nationwide, reflecting the importance of closely monitoring data and having a flexible system. The federal level EPI team was very involved in the activities at the district and health facility levels, including review and approval of all district micro-plans, regular on-site supervisory visits, and monthly calls to all districts to ensure adequate vaccine and syringe supplies. Participation of state level staff in regular meetings at the federal level also assured districts stayed on track to reach targets. When mid-year poorly performing districts were identified, acceleration activities were planned. The DQS process has been internalized and state level supervisors and most LOs use the DQS as a supervisory tool to collect comprehensive data down to the district level and actively monitor these data.

North Sudan should continue to work on improving the immunization programme through RED to meet and exceed the Global Immunization Vision and Strategy goals of $90 \%$ coverage nationally and at least $80 \%$ coverage in all districts [1]. Although national DPT3 coverage reached $90 \%$ coverage in 2006, measles coverage lagged at $78 \%$ nationally and only $44 \%$ of districts achieved $\geq 80 \%$ measles coverage in 2006. Additional efforts are needed to boost measles coverage in order to meet the WHO Regional goal of measles elimination by 2010 and ensure that all districts have $>80 \%$ DPT3 coverage.

\section{Acknowledgements}

We would like to thank the WHO Sudan country office and Dr Salah Haithami for facilitating and supporting this evaluation and the WHO Regional Office for the Eastern Mediterranean for logistical and financial support. We would also like to thank all the EPI staff in North Sudan who answered all of our questions and provided data, and Dr Fazal Ather, Dr Ahmed Hardan Ismael, Dr Abdel Mageid Mohammed El Amin and D. Hythum Massed for assistance in case study data collection.

This work was funded by the United States Centers for Disease Control and Prevention with additional support from the WHO Regional Office for the Eastern Mediterranean.

\section{Author note}

The findings and conclusions in this report are those of the authors. We declare no conflicts of interest.

\section{References}

1. Global Immunization Vision and Strategy (GIVS) [webpage]. (http://www.who.int/immunization/givs/en/index.html, accessed 5 October 2011).

2. World Health Organization African Region. Implementing the Reaching Every District Approach: A guide for District Health Management Teams. (http://www.who.int/immunization_delivery/systems_policy/AFRO-RED-guide_2008.pdf, accessed 5 October 2011).

3. Global Polio Eradication Initiative: 2005 annual report. Geneva, World Health Organization, Rotary International, Centers for Disease Control and Prevention, United Nations Children's Fund, 2006 (WHO/Polio/06.02)

4. Ryman $\mathrm{T}$ et al. Reaching every district (RED) approach to strengthen routine immunization services: evaluation in the African region, 2005. Journal of Public Health, 2010, 32(1):1825.
5. El Zein HA et al. Rehabilitation of the Expanded Programme on Immunization in Sudan following a poliomyelitis outbreak. Bulletin of the World Health Organization, 1998, 76(4):335-341.

6. The immunization data quality self-assessment (DQA) tool. Geneva, Word Health Organization, 2005 (http://www.who.int/ immunization_monitoring/routine/DQS_tool.pdf, accessed 5 October 2011).

7. Burton, A. WHO and UNICEF estimates of national infant immunization coverage: methods and processes. Bulletin of the World Health Organization, 2009, 87(7):535-541. 